\section{DIN EN ISO 15193}

G. Schumann

Hannover, Deutschland

\section{Englischer Begriff ISO 15193}

Beschreibung Die Internationale Norm „In-vitroDiagnostika - Messung von Größen in Proben biologischen Ursprungs - Anforderungen, Inhalt und Aufbereitung von Referenzmessverfahren" enthält Anforderung an Referenzmessverfahren, die auch als „Messverfahren höherer Ord- nung" bezeichnet werden. Solche Verfahren und Referenzmaterialien ( $\triangleright$ DIN EN ISO 15194) sind die wichtigsten Bestandteile von Referenzsystemen für medizinische Laboruntersuchungen und zeichnen sich durch große Genauigkeit und kleine Messunsicherheit aus.

\section{Literatur}

DIN EN ISO 15193 (2009) In-vitro-Diagnostika - Messung von Größen in Proben biologischen Ursprungs - Anforderungen, Inhalt und Aufbereitung von Referenzmessverfahren. Beuth-Verlag, Berlin 